\title{
Massimo Colesanti, Il Mistero Stendhal. Saggi, note, confronti
}

\section{Annalisa Bottacin}

\section{(2) OpenEdition}

1 Journals

\section{Edizione digitale}

URL: http://journals.openedition.org/studifrancesi/4394

DOI: $10.4000 /$ studifrancesi.4394

ISSN: 2421-5856

\section{Editore}

Rosenberg \& Sellier

\section{Edizione cartacea}

Data di pubblicazione: 1 settembre 2016

Paginazione: 338-341

ISSN: 0039-2944

\section{Notizia bibliografica digitale}

Annalisa Bottacin, "Massimo Colesanti, II Mistero Stendhal. Saggi, note, confronti », Studi Francesi

[Online], 179 (LX | II) | 2016, online dal 01 septembre 2016, consultato il 18 septembre 2020. URL :

http://journals.openedition.org/studifrancesi/4394 ; DOI : https://doi.org/10.4000/studifrancesi.4394

Questo documento è stato generato automaticamente il 18 settembre 2020.

\section{(c) (i) (9)}

Studi Francesi è distribuita con Licenza Creative Commons Attribuzione - Non commerciale - Non opere derivate 4.0 Internazionale. 


\title{
Massimo Colesanti, Il Mistero Stendhal. Saggi, note, confronti
}

\author{
Annalisa Bottacin
}

\section{NOTIZIA}

MASSIMO COLESANTI, Il Mistero Stendhal. Saggi, note, confronti. Roma, Edizioni di Storia e Letteratura, «Quaderni di Cultura Francese a cura della Fondazione Primoli» 49, 2015, 208 pp., ill.

1 L'uscita di questo corposo volume, arricchito di un'iconografia che riproduce le raffinate caricature del principe Filippo Caetani, amico di Stendhal, designa in campo critico-letterario l'avvio di un processo atto a orientare l'indagine verso i meccanismi più sottili dell'atto creativo e si concentra su un'interrogazione intesa a portare lo sguardo su varie figure che vengono qui ri-scoperte, ri-visitate, in una riflessione che ha come movimento Stendhal - per lo più lo Stendhal romano - ma, che per essere percorsa, necessita di una densità di sguardi e di spostamenti, intesi a insinuarsi nella leggenda stendhaliana, qui interpretata con acutezza, profondità e grande intuizione da un autorevole studioso, quale è Massimo Colesanti. Che cos'è il testo stendhaliano? Cosa significa designare o nominare in tal modo un testo in cui si innestano vari tipi di scrittura con la discontinuità, le fratture, le riprese, ma altresì i segreti, i plagi, la sferzante ironia, tipici della penna di Henri Beyle? Massimo Colesanti conduce il lettore nei meandri più intimi di quel «mistero» avvolgente e assoluto, il cui riflesso si espande in pagine tanto modulate e ricche di fascino, perché intimamente legate al gusto di sorprendere. E ancor più è da sottolineare che un'altra peculiarità di questa pubblicazione, che vede la riunione in volume di saggi precedentemente pubblicati in riviste - eccetto Melodramma alla Locanda Serny - è quella di fare partecipe il lettore meno intriso di Stendhal (quindi non solo l'amateur o gli addetti ai lavori) in una strategia capace di moltiplicare le occasioni di contatto con un autore, come ben rileva il critico nella Premessa (pp. IX-XII), confinato in spazi talvolta difficili o vaghi, che nel 
volume vengono a fissarsi per ricreare un nuovo rapporto privilegiato con un autore prediletto, quale è per Colesanti il genio grenoblese.

2 Il Mistero Stendhal si compone di diciotto contributi, seguiti da una corposa Appendice (Fortuna o sfortuna di Stendhal, pp. 173-92); se, come precedentemente segnalato, è per lo più un Henri Beyle «romano» quello che attraversa le pagine, l'omaggio all'amata Milano, spazio scenografico e affettivo d'elezione, entra in gioco sin dal primo testo, con una riflessione inerente L"Incipit" della "Chartreuse de Parme" (pp. 1-11), un romanzo in cui la presenza di Napoleone nella prima campagna d'Italia fa da «vera e propria ouverture» all'opera. In effetti, evidenzia lo studioso, il primo capitolo, che è l'unico ad avere un titolo, Milan en 1796, è un inizio che «si salda [...] alla rievocazione d'una palingenesi storica, epocale, ma che coinvolge nella sua dinamica [...] lo stesso scrittore, la stessa felicità inventiva della sua parola» (p. 3). In una comparazione con gli incipit di altri romanzi, quali Armance, Lucien Leuwen o Le Rouge et le Noir, l'A. esalta le particolarità e l'estrema forza narrativa di un'opera delle infinite letture, reputata da molti come il più bel romanzo del nostro Risorgimento, o romanzo politico o romanzo d'amore, di passioni e d'avventura o romanzo puro; per il critico, «[1]a Chartreuse è tutte queste cose insieme senza esserne esclusivamente nessuna. Anche perché è una straordinaria metafora o allegoria, favolosa e realistica a un tempo, dell'esistenza, delle vicende umane in un'altrettanto straordinaria costruzione della scrittura» (p. 10). L'investitura di Milano a città prescelta per «vivere e morire» è legittimata da Colesanti attraverso l'analisi delle lettere inviate alla prediletta sorella dalla città lombarda tra il 1800 e il 1813, sin dalla prima folgorazione con l'amata Italia ( $C$ Chez moi»: Milano nelle lettere a Pauline, pp. 13-21). Sin dalla prima missiva, quella del 29 giugno 1800, si percepisce subito la grande attrazione di Henri per la città mitica che in un certo senso è oggetto del sogno, figura del desiderio; si tratta di uno scritto che «indubbiamente [è] documento di grande importanza, considerando che per questo periodo non abbiamo altri riscontri (il Journal inizia un anno dopo, il 18 aprile 1801)»(p. 15). Ricordo profondo, quello di Milano, che si ravviva molti anni più tardi nelle pagine dalla Vie de Henry Brulard, situando «questi primi mesi passati a Milano (e dintorni) come del più bel tempo della sua vita», ma lo furono veramente, si chiede lo studioso? Non fu forse esagerata la nascita del mito di Milano «come patria ideale?». Forse all'inizio, ma per gli anni successivi le cose in parte mutarono. Il terzo saggio, Arrigo Beyle "romano". Una noia feconda (pp. 23-32) ripercorre i cammini milanesi di Stendhal, delineandone i contenuti, evidenziando che il rapporto con la Città Eterna non fu certo meno profondo, seppur molto diverso; lo scrittore vi si era recato quattro volte prima di essere trasferito dalla sede consolare triestina a quella civitavecchiese, ne aveva parlato in Rome, Naples et Florence e aveva prodotto le Promenades dans Rome. Quella «noia» tanto decantata forse non fu così reale come pare, suggerisce il critico; a Roma, frequenta i salotti più illustri come quelli dei Cini, dei Potenziani, dei Torlonia e dei Caetani, ma anche famiglie borghesi, pratica i teatri, ebbe «alcune avventure di passaggio» (p. 27) e alcuni amori tra cui quello infelice e impossibile con Giulia Cini. «È una noia feconda, creativa» (p. 29), in quanto, in quegli anni, Stendhal produsse pagine memorabili per cui «il suo lungo periodo romano si chiude con un bilancio fortemente attivo dal punto di vista della creazione letteraria, e di un peso specifico fortemente superiore alla tara, in ogni senso, della noia, e dei tanti momenti di malumore» (pp. 31-32). Quale Stendhal si cela nel quarto testo, dal titolo Giocando a Roma a «nasconnarella» (pp. 33-41), tanto da «ritornare ragazzo tanto vivace e discolo (quale del resto, sotto tanti aspetti, è un po' sempre rimasto) per giocare a rimpiattino nelle piazzette, nelle viuzze e nei vicoli della 
città»? (p. 33). Senza dubbio alcuno, rileva l'A., la questione appare quanto mai manifesta nelle pagine delle Promenades dans Rome, un'opera più volte ripresa in mano dall'autore, in cui sono ricorrenti quei percorsi ludici stendhaliani riconoscibili dalle segrete e quasi indecifrabili note, dalle postille, dai titoletti e dagli indici, spesso soppressi, dagli asterischi, «altra forma di reticenza o di maschera» (p. 39), che sono parte integrante del narrato, quasi che un atteggiamento intrigante ma raffinatissimo devii volutamente per meglio dissimulare, e talvolta si ritragga per poi subito riproporsi in geniali giochi di specchi, fascinosi e insieme insinuanti. Da un'allargata discussione avvenuta a Roma a Palazzo Lancellotti, in cui aveva abitato Chateaubriand, allorché era stato segretario d'ambasciata del cardinale Fesch, relativa a Stendhal e all'autore dei Mémoires d'Outre-Tombe, dove Colesanti si era trovato presente, l'eminente critico trae questo apporto (Gatti vaticani e cani bastardi, pp. 43-49) inerente a quale dei due scrittori abbia meglio rappresentato la Roma di Pio VII e di Leone XII. I due «contendenti - ricorda Colesanti - erano due grossi personaggi della cultura di mezzo secolo fa, un intellettuale cattolico di spicco e sacerdote, e un famoso stendhaliano: Don Giuseppe De Luca e Vittorio Del Litto » (p. 43). Naturalmente ognuno dei due teneva alta la propria posizione, ma fu Don Giuseppe ad avere l'ultima parola, asserendo che lo Stendhal «console» era senza alcun dubbio inferiore all'ambasciatore Chateaubriand. Certo si trattava di una battuta, che comunque si rivelava assai veritiera, sulla quale lo studioso inizia una profonda digressione che conduce a un parallelo tra i due scrittori, con varie esemplificazioni tratte dai Mémoires e dalle Promenades con riferimenti a (raffinati) gatti e a cani (bastardi), questi ultimi molto amati dal grenoblese che a Civitavecchia ne ha avuti almeno due. Di notevole interesse per il tema, che portò anche a una piccola querelle in Stendhalie, il contributo I Pretendenti delusi (pp. 51-56), che ripercorre l'amore del grenoblese per Giulia Rinieri de' Rocchi, sulla cui figura scrissero Luigi Foscolo Benedetto e critici eminenti, quali Boyer, Trompeo e Michel. In occasione del congresso stendhaliano di Roma del 1983, riporta Colesanti di essere stato avvicinato da un distinto gentiluomo toscano che chiedeva la parola, e fu proprio lo stesso critico a rivolgersi al presidente di turno della seduta, l'illustre studioso Jean Rousset, chiedendogli di invitare a parlare Lapo Rinieri de' Rocchi, questo il suo nome che, insieme a Giannatonio Stegagno, qualche anno dopo, e precisamente nel 1987, avrebbe dato alle stampe il frutto di attente ricerche archivistiche, con il titolo Storia di Giulia. Si tratta di una documentazione abbondante per lo più inedita, che mette a nudo il carattere, il comportamento, la viva intelligenza e la modernità di questa nobile senese che disse a Stendhal di amarlo e che lo scrittore profondamente amò in una relazione segreta. In effetti, rileva l'A. «[d]a tutti i carteggi dai quali Rinieri e Stegagno han saputo estrarre e intrecciare, con grande misura e perizia, tutto ciò che veramente poteva riuscire utile a ritessere ed a completare il tema che si erano proposti [...] è la figura di Giulia a grandeggiare, e a testa alta...» (p. 55). Sull'avvincente problematica del ritratto di Stendhal, che avrebbe posseduto il conte Primoli nel suo palazzo romano e che alcuni studiosi «hanno visto o meglio creduto di osservare come tale», si incentra il settimo contributo dal titolo Un ritratto fantasma? Quartum non datur (pp. 57-64), seguito da un altro testo di argomento romano, riferito questa volta al conte Filippo Caetani, corredato dalla riproduzione di una serie di caricature di sua mano, tra cui una presunta del console, a matita e acquerello su cartoncino (Don Filippo, l'amico romano, pp. 65-74). Colesanti esordisce ricordando la sua collaborazione a un'importante mostra, Il salotto delle caricature, allestita a Roma al Museo Napoleonico nel 1999; l'introduzione al catalogo è firmata da Luigi Fioriani e s'intitola Filppo Caetani, l'ironia nella Roma papale 
dell'ottocento, da ritenersi «l'unico studio da lui dedicato a questa figura indubbiamente minore, ma a torto quasi del tutto trascurata fino ad allora» (p. 65). Lo studioso ben evidenzia la figura del principe e in particolar modo il suo profondo rapporto con il grenoblese, che fu legato anche al fratello Michelangelo. Una frequentazione assidua che comunque non trova riscontro nella corrispondenza, il che non può non stupire; nell'Archivio Caetani non vi è traccia di alcuno scambio epistolare tra i due amici, infatti «nulla o quasi è stato ritrovato finora direttamente dalla parte di Filippo, né note, né allusioni, né lettere a Stendhal» (p. 69). Nella mappa che Stendhal traccia per il suo periplo in Sicilia, che rimarrà comunque un voyage imaginaire, due circoletti localizzano il cratere dell'Etna; in effetti l'immagine del vulcano, più che mai assurto a funzione estetica, investe pienamente lo scrittore, come è ben evidenziato nel saggio dal titolo Trittico napoletano (pp. 75-86), in cui l'insigne studioso, prendendo spunto dalla lettera invita da Beyle a Domenico Fiore il 14 gennaio 1832, ne stralcia «tre temi episodici o aspetti napoletani [...]: la scalata al Vesuvio, Pompei, e un (presunto) busto di Tiberio, di marmo» (p. 75). L'andare in cerca $e$ verso qualcosa di terrificante e misterioso, quale un vulcano in eruzione, è, a dire di Colesanti, un viaggio verso un luogo dalle grandi probabilità di sorpresa, che superando la razionalità rappresentativa della carta topografica trova l'articolazione più segreta nell'immaginario. Il decimo intervento è dedicato a Courier e Custine nelle Calabrie napoleoniche (pp. 87-98), due scrittori che vennero in Italia nel 1811, allorché Stendhal visitava il nostro paese. PaulLouis Courier aveva partecipato a varie campagne militari in Italia e vi era rimasto per $\mathrm{i}$ suoi studi di grecista. Il giovane Astolphe de Custine vi era invece giunto con la madre, il precettore e il dottor Koreff con il preciso intento di visitare in particolar modo le Calabrie. Colesanti spiega il motivo per cui, tra i tanti viaggiatori francesi che hanno lasciato relazioni di viaggio in Italia, abbia proprio scelto questi due letterati, in quanto «partendo da situazioni psicologiche e da esperienze concrete assai diverse, anzi per molti aspetti contrarie, mostrano alcune coincidenze significative di osservazioni e di giudizi sugli uomini, sulle cose, sugli eventi italiani di quegli anni» (pp. 87-88). I rapporti intercorsi tra Custine, Stendhal e Balzac, per altro poco trattati dalla critica, sono accuratamente approfonditi nell'undicesimo studio (Custine fra Stendhal e Balzac, pp. 99-107). Grazie a varie esemplificazioni estrapolate per lo più dai carteggi, Colesanti evidenzia l'importanza non solo dei rapporti mondani o degli incontri anche casuali avvenuti fra i tre scrittori, ma propone osservazioni e suggerimenti sui loro scambi epistolari. Il dodicesimo contributo, Il conte Primoli, Belli e Stendhal (pp. 109-116) trae spunto dall'edizione integrale dei tre viaggi del Belli a Milano, che fu presentato alla Fondazione Primoli, di cui Colesanti è l'attuale Presidente. Ci si è a lungo chiesti se vi fosse stata una reale conoscenza del grenoblese con il poeta romanesco, finché Pietro Paolo Trompeo ne trovò in una terzina italiana del poeta un accenno, in un contesto in cui il Belli esalta ancora una volta le doti sceniche dell'attrice drammatica Amalia Bettini, cui dedicò vari sonetti, come ricordava lo stesso Trompeo nel suo bel saggio, Amalia B. Il viaggio milanese del Belli, redatto in francese, seppur un francese non sempre corretto, crea alcune perplessità in Colesanti che si chiede il perché di questa scelta e ne formula alcune interessanti ipotesi. Legato ancora al tema del Belli il tredicesimo contributo dal titolo La Roma e il Belli di Sainte-Beuve. Stendhal fra le quinte (pp. 117-28) che ripropone un celebre episodio legato al viaggio che Sainte-Beuve fece in Italia e "per la visione che egli ebbe di Roma» (p. 117). Nel viaggio di ritorno, lo scrittore incontra il 20-21 giugno 1839, sul battello che da Civitavecchia lo riporta a Marsiglia, Gogol' che disquisisce con entusiasmo su un poeta che scrive in dialetto 
«trasteverino», appunto il Belli. Nel taccuino, in cui Sainte-Beuve annota alcuni tratti del suo viaggio a Napoli e a Roma, vi è un appunto sul poeta vernacolare; ma Colesanti non tralascia la posizione di Gogol' nei riguardi della penisola, arricchendo queste interessanti pagine di molte, approfondite curiosità, per altro poco note. Gigi Huetter stendhaliano in campo (pp. 129-135) è un ritratto dell'insigne epigrafista ed erudito "romanista", le cui lettere inviate a Pietro Paolo Trompeo sono conservate negli archivi della Fondazione Primoli. Riportate alla luce da Colesanti rievocano ricordi, allusioni a lavori che Trompeo lesse, risposte scherzose e allusioni allo stesso Stendhal. Purtroppo, conclude l'insigne critico, non siamo in possesso delle risposte di Trompeo a Huetter, che condurrebbero senza dubbio alcuno a riflessioni intorno a Stendhal. Il quindicesimo studio è dedicato alla pubblicazione, nel 2012, del Rosso e Nero nella traduzione di Aldo Palazzeschi, volume curato da Francesca Mecatti ("Rosso e Nero" di Palazzeschi, pp. 137-143). Analizzando in profondità questa traduzione, fino allora rimasta inedita, Colesanti ne evidenzia l'orientamento sovente «"anormale", sui generis, per molti e complessi motivi e aspetti» (p. 140), elogiando la curatrice per il rigoroso lavoro svolto. Sciascia e il mistero Stendhal (pp. 145-154), sedicesimo contributo di questa appassionante raccolta, evidenzia da un lato la devozione e l'amicizia per il grande scrittore siciliano da parte dell'autore e dall'altro «l'adorazione per Stendhal» che Sciascia coltivò per tutta la sua esistenza, lasciando pagine memorabili, raccolte da Maria Andronico Sciascia, e con un saggio dello stesso Colesanti, in una bella edizione apparsa nel 2003. Finissimo letterato, autore di alcuni tra i capolavori della narrativa italiana e raffinato e vigile intellettuale, Sciascia viene tra l'altro ricordato da Colesanti quale assiduo lettore «di una galleria di scrittori [francesi] non tutti omogenei fra loro, certo, ma a lui congeniali e conformi e tutti però impegnati nelle loro posizioni ideologiche, nelle loro polemiche, nella loro dissidenza con le imposture e le tartuferie del secolo in cui sono vissuti e in cui tuttavia hanno voluto agire e incidere, per la giustizia, per la verità, per la libertà» (p. 145). Da Montaigne a Pascal, a Gide, a Bernanos a Valery Larbaud, ma anche ai memorialisti e agli scrittori tra i più amati da Stendhal. Dai suoi scritti su Henri Beyle, nota Colesanti, si evince quanto Sciascia abbia amato «la profondità e la modernità, il brio della scrittura di Stendhal» (p. 148), intuendone intimamente i lati più segreti. Assai degno di nota per l'argomento trattato, il diciassettesimo saggio (L'amico siciliano: il «pazzo» Micciché e il suo doppio, pp. 155-165) che fa emergere dati innovativi e preziosissimi sulla figura di un amico di Stendhal esule a Parigi, Michele Palmieri di Micciché, dalla «personalità contraddittoria, mobile, piena di umori e di risentimenti, e in fondo caparbia, esosa, vincolata a ricordi e pregiudizi, pur nella sua avventurosa dissipazione libertina, e nella sua apparente svagatezza» (p. 155). Fu autore di vari scritti e alcuni riproposti recentemente, motivo anche questo dell'interesse di Colesanti per il nobile siciliano. Il critico evidenzia acutamente le affinità tra Stendhal e Miccichè, con una serie di esemplificazioni tratte da pagine stendhaliane che, messe a raffronto con atteggiamenti ed episodi relativi all'esistenza e ai testi del fuoriuscito, si rivelano determinanti. Pubblicato per la prima volta in questa raccolta, il Melodramma alla Locanda Serny (pp. 165-169) prende spunto da un autorevole romanzo di Marco Fabio Apolloni, Il mistero alla locanda Serny, uscito nel 2003 e finalista al premio Strega. Tra i personaggi " ritroviamo Stendhal» scrive Colesanti, insieme alla "grande avventura napoleonica» e alla ritirata di Russia, e non è quindi difficile «stabilire un immediato collegamento con gl'interessi degli studiosi stendhaliani o napoleonici» (p. 165). Il romanzo di notevole pregio stilistico e narrativo, dal taglio in parte fantastique e non solo poliziesco, reca anche un sottotitolo: Roma, 1839. 
Romanzo a quattro voci con artisti, delitti, sparizioni e magie. Un sottotitolo intrigante che crea, nell'acuta lettura di Colesanti, ampie soglie di apertura verso circuiti emotivi su cui riflettere. Tra l'altro, nella locanda Serny, il grenoblese prese alloggio nelle giornate romane e ne fu sempre soddisfatto al punto di raccomandarla agli amici. L'importante volume presenta in Appendice un corposo saggio che ha segnato fortemente la critica stendhaliana, Fortuna o sfortuna di Stendhal? (pp. 173-192), lavoro che abbraccia non solo la grande figura di Henri Beyle, ma accompagna il lettore a seguirne la storia, analizzando la «fortuna» e la «sfortuna» di un autore immenso in quanto, scrive l'eminente critico, «[i]l discorso, così ampio e ramificato su di lui, è sempre un continuo punto e a capo, pieno di riprese, di svolte, di ritorni per altre vie e in altre direzioni» (p. 192). Un'accurata ed esaustiva Nota bibliografica (pp. 193-196) seguita dall'Indice dei nomi, dall'Indice delle opere e dall'Indice dei luoghi (pp. 197-208) conclude un insieme di autorevoli studi in cui si evidenziano altresì tracce di silenzi, di vuoti di parola, di ciò che è nascosto, taciuto o mascherato. Al pari del «mistero» Stendhal. 